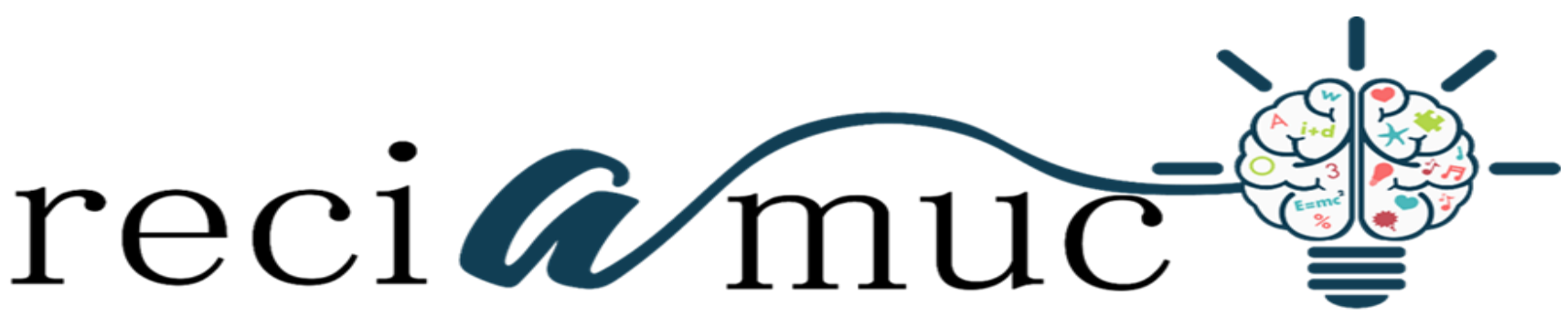

Revista cientifica de investigación actualización del mundo de las ciencias

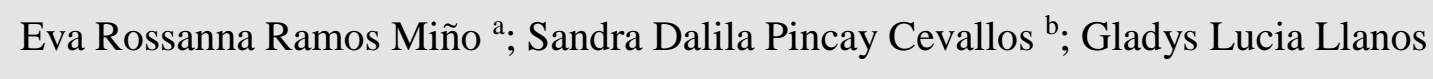
González $^{\text {c; }}$ Celia Matilde Vinueza Villacis ${ }^{\mathrm{d}}$

Estimulación temprana sinónimo de un mejor desarrollo infantil

Early stimulation synonymous with better child development

Revista Científica de Investigación actualización del mundo de las Ciencias. Vol. 3 núm., 1, enero, ISSN: 2588-0748, 2018, pp. 164-180

DOI: $10.26820 /$ reciamuc/3.(1).enero.2019.1164-180

URL: $\underline{\text { http://reciamuc.com/index.php/RECIAMUC/article/view/228 }}$

Código UNESCO: 3205 Medicina Interna

Tipo de Investigación: Artículo de Revisión

Editorial Saberes del Conocimiento

Recibido: 10/12/2018

Aceptado: 20/01/2019

Publicado: 30/01/2019

Correspondencia: director@reciamuc.com
a. Médico; Guayaquil, Ecuador.
b. Médico; Guayaquil, Ecuador.
c. Médico; Guayaquil, Ecuador.
d. Médico; Guayaquil, Ecuador. 


\section{Estimulación temprana sinónimo de un mejor desarrollo infantil}

Vol. 3, núm. 1., (2019)

Eva Rossanna Ramos Miño; Sandra Dalila Pincay Cevallos; Gladys Lucia Llanos González; Celia Matilde Vinueza Villacis

\section{RESUMEN}

El proceso investigativo estudia la efectividad que tiene la estimulación temprana durante el desarrollo infantil en los primeros cinco años de vida y los beneficios que aporta en la etapa adolescente y adulta de los seres humanos, a través, de una revisión bibliográfica que proporciona información importante acerca de temas referentes a la estimulación temprana y que brindaron aportes significativos a la investigación. El trabajo hace mención, a las actividades que se deben tomar en consideración para el desarrollo de los niños y como los ayuda a potenciar los procesos cerebrales e impulsar las funciones cognitivas, lingüísticas, motriz y social, teniendo en cuenta, que el éxito de la estimulación temprana en personas adolescentes y adultas está ligado a factores como la nutrición y los niveles socio económicos, por lo que se cree necesario, la implementación de centros o instituciones tanto públicos como privados que promuevan las tareas y actividades de estimulación para que toda la población tenga acceso y puede ser guiada y para poder aplicarlo a los bebes en los hogares. La estimulación debe comenzar en los primeros días de nacido, por medio de, juegos y actividades que se especifican en el desarrollo del trabajo de acuerdo a la edad del niño, tomando en consideración que el Coeficiente Intelectual de los individuos en la adolescencia está ligado a la estimulación temprana de acuerdo a un estudio realizado en donde se pudo determinar que aquellos adolescentes que fueron estimulados a temprana edad mostraron un CI mayor que aquellos que no fueron estimulados.

Palabras Claves: Estimulación Temprana; Desarrollo Infantil; Coeficiente Intelectual; Actividades; Juegos; Niños. 


\title{
Estimulación temprana sinónimo de un mejor desarrollo infantil
}

Vol. 3, núm. 1., (2019)

Eva Rossanna Ramos Miño; Sandra Dalila Pincay Cevallos; Gladys Lucia Llanos González; Celia Matilde Vinueza Villacis

\begin{abstract}
The research process studies the effectiveness of early stimulation during childhood development in the first five years of life and the benefits it brings in the adolescent and adult stage of human beings, through a bibliographic review that provides important information about of topics related to early stimulation and that provided significant contributions to research. The work makes mention of the activities that must be taken into consideration for the development of children and how it helps to boost brain processes and promote cognitive, linguistic, motor and social functions, taking into account that the success of the Early stimulation in adolescents and adults is linked to factors such as nutrition and socio-economic levels, which is why it is necessary to implement public or private centers or institutions that promote stimulation tasks and activities so that the entire population have access and can be guided and to be able to apply it to babies in homes. The stimulation should begin in the first days of birth, by means of, games and activities that are specified in the development of the work according to the age of the child, taking into consideration that the Intellectual Coefficient of the individuals in adolescence is linked to early stimulation according to a study conducted where it could be determined that those adolescents who were stimulated at an early age showed a higher IQ than those who were not stimulated.
\end{abstract}

Key Words: Early stimulation; Child development; Intelligence quotient; Activities; Games; Children. 


\section{Estimulación temprana sinónimo de un mejor desarrollo infantil}

Vol. 3, núm. 1., (2019)

Eva Rossanna Ramos Miño; Sandra Dalila Pincay Cevallos; Gladys Lucia Llanos González; Celia Matilde Vinueza Villacis

\section{Introducción.}

La estimulación temprana representa una de las actividades más importantes en los primeros años de vida, ya que, en esta etapa se maduran y desarrollan capacidades fundamentales entre las que se encuentran: el área del lenguaje, sensorial, física y psicológica. Este proceso se recomienda comenzarlo en los primeros 45 días de nacido, a través, de programas adaptados a la estimulación temprana. Este tipo de actividades va enfocado a una serie de juegos y ejercicios realizados en un ambiente adecuado y específico para cada edad.

Por esta razón, la importancia de la estimulación y el desarrollo en la primera etapa de la infancia y la educación no debe ser subestimada. Estudios han demostrado que los niños que participaron en programas de educación tenían más probabilidades de matricularse en la educación primaria y eran más interesados en aprender habilidades académicas, como matemáticas, escritura y lectura; que su compañeros que no participaron en dichos programas.

En los países subdesarrollados un gran número de niños están expuestos a múltiples factores de riesgo en los primeros años de vida, incluida la mala salud, la desnutrición y los bajos niveles de estimulación en el hogar, entre otros. Los factores de riesgo traen consigo escenarios de pobreza caracterizados por una baja educación materna, crianza de adolescentes, desnutrición infantil, bajo peso al nacer, altos niveles de depresión materna, barrios inseguros y bajos niveles de estimulación en el hogar.

El autor (Wachs, 2000) expresa que el desarrollo infantil es multidimensional e incluye dominios cognitivo-lenguaje, sensorio-motor y socio-emocional, los cuales son interdependientes. Sin embargo, existe un creciente reconocimiento de la importancia de los 


\section{Estimulación temprana sinónimo de un mejor desarrollo infantil}

Vol. 3, núm. 1., (2019)

Eva Rossanna Ramos Miño; Sandra Dalila Pincay Cevallos; Gladys Lucia Llanos González; Celia Matilde Vinueza Villacis

dominios no cognitivos en la predicción del rendimiento académico, la productividad y el funcionamiento social de los niños en la edad adulta.

El desarrollo infantil también está determinado por la influencia de una interacción entre la herencia genética de los niños, es decir, el estado biológico por ejemplo, estado de salud y nutrición, la calidad de la interacción materno-infantil y por las características del vecindario o medio ambiente en el que se desenvuelve el infante (Wachs 2000).

Durante los primeros años de vida ocurre un rápido crecimiento y desarrollo en todos los dominios del ser humano, formando una base sólida para el aprendizaje de habilidades posteriores. El autor (Heckman, 2006) opina que los niños que viven en entornos desfavorecidos tienen más probabilidad de estar expuesto a riesgos biológicos y ambientales que afectan el crecimiento del cerebro y pueden conducir a cambios duraderos en la estructura y función del cerebro en desarrollo.

De los cuatro a los seis años, han surgido brechas persistentes en el desarrollo de los niños tanto en lo cognitivo como en lo no cognitivo, dominios entre los niños de entornos desfavorecidos y sus más favorecidos compañeros. De acuerdo a esto los estudios arrojan que existe una base de evidencia sustancial que muestra que la exposición a personas desfavorecidas los entornos durante los primeros años de vida se asocian con muchos resultados negativos en la adolescencia y la edad adulta, que incluyen un coeficiente intelectual y un rendimiento académico más bajos, aumentos en los estudios antisociales comportamiento y menores ganancias en la edad adulta (Heckman J. \&., 2007). 


\section{Estimulación temprana sinónimo de un mejor desarrollo infantil}

Vol. 3, núm. 1., (2019)

Eva Rossanna Ramos Miño; Sandra Dalila Pincay Cevallos; Gladys Lucia Llanos González; Celia Matilde Vinueza Villacis

La implementación de instituciones que se aboquen a fortalecer los ejercicios y juegos de estimulación temprana, es una de las tareas por parte de cada país, si se trabaja desde el nivel inicial y que sea de acceso a todo tipo de público, se genera una corriente de niños más avanzados que muestren desarrollos en todas las áreas y que aseguren un mayor aprendizaje en la vida adulta. Para ellos se debe promover el funcionamiento de guarderías tanto públicas como privadas con el fin de que toda la población tenga acceso incentivando a su vez la estimulación en el hogar.

Mediante la redacción de este trabajo investigativo se pretende promover la utilización de actividades de estimulación temprana con el fin de impulsar los procesos mentales del cerebro del niño potenciando las funciones cognitivas, lingüísticas, motriz y social.

\section{Métodos y materiales.}

Para el desarrollo de este proceso investigativo, se plantea como metodología la encaminada hacia una orientación científica particular que se encuentra determinada por la necesidad de indagar en forma precisa y coherente una situación, en tal sentido (Dávila, 2015) define la metodología "como aquellos pasos previos que son seleccionados por el investigador para lograr resultados favorables que le ayuden a plantear nuevas ideas".(p.66)

Lo citado por el autor, lleva a entender que el desarrollo de la acción investigativa busca simplemente coordinar acciones enmarcadas en una revisión bibliográfica con el fin de complementar ideas previas relacionadas Estimulación temprana sinónimo de un mejor 


\section{Estimulación temprana sinónimo de un mejor desarrollo infantil}

Vol. 3, núm. 1., (2019)

Eva Rossanna Ramos Miño; Sandra Dalila Pincay Cevallos; Gladys Lucia Llanos González; Celia Matilde Vinueza Villacis

desarrollo infantil, a través de una revisión de literatura, para así finalmente elaborar un cuerpo de consideraciones generales que ayuden a ampliar el interés propuesto.

\section{Tipo de Investigación}

Dentro de toda práctica investigativa, se precisan acciones de carácter metodológico mediante las cuales, se logra conocer y proyectar los eventos posibles que la determinan, así como las características que hacen del acto científico un proceso interactivo ajustado a una realidad posible de ser interpretada. En este sentido, se puede decir, que la presente investigación corresponde al tipo documental, definido por Castro (2016), "se ocupa del estudio de problemas planteados a nivel teórico, la información requerida para abordarlos se encuentra básicamente en materiales impresos, audiovisuales y /o electrónicos”. (p.41).

En consideración a esta definición, la orientación metodológica permitió la oportunidad de cumplir con una serie de actividades inherentes a la revisión y lectura de diversos documentos donde se encontraron ideas explicitas relacionadas con los tópicos encargados de identificar a cada característica insertada en el estudio. Por lo tanto, se realizaron continuas interpretaciones con el claro propósito de revisar aquellas apreciaciones o investigaciones propuestas por diferentes investigadores relacionadas con el tema de interés, para luego dar la respectiva argumentación a los planteamientos, en función a las necesidades encontradas en la indagación.

\section{Fuentes Documentales}

El análisis correspondiente a las características que predomina en el tema seleccionado, llevan a incluir diferentes fuentes documentales encargadas de darle el respectivo apoyo y en ese 


\section{Estimulación temprana sinónimo de un mejor desarrollo infantil}

Vol. 3, núm. 1., (2019)

Eva Rossanna Ramos Miño; Sandra Dalila Pincay Cevallos; Gladys Lucia Llanos González; Celia Matilde Vinueza Villacis

sentido cumplir con la valoración de los hechos a fin de generar nuevos criterios que sirven de referencia a otros procesos investigativos. Para (CASTRO, 2016) las fuentes documentales incorporadas en la investigación documental o bibliográfica, "representa la suma de materiales sistemáticos que son revisados en forma rigurosa y profunda para llegar a un análisis del fenómeno".(p.41). Por lo tanto, se procedió a cumplir con la realización de una lectura previa determinada para encontrar aquellos aspectos estrechamente vinculados con el tema, con el fin de explicar mediante un desarrollo las respectivas apreciaciones generales de importancia.

\section{Técnicas para la Recolección de la Información}

La conducción de la investigación para ser realizada en función a las particularidades que determinan a los estudios documentales, tiene como fin el desarrollo de un conjunto de acciones encargadas de llevar a la selección de técnicas estrechamente vinculadas con las características del estudio. En tal sentido, (Bolívar, 2015), refiere, que es "una técnica particular para aportar ayuda a los procedimientos de selección de las ideas primarias y secundarias”. (p. 71).

Por ello, se procedió a la utilización del subrayado, resúmenes, fichaje, como parte básica para la revisión y selección de los documentos que presentan el contenido teórico. Es decir, que mediante la aplicación de estas técnicas se pudo llegar a recoger informaciones en cuanto a la revisión bibliográfica de los diversos elementos encargados de orientar el proceso de investigación. Tal como lo expresa, (Bolivar, 2015) "las técnicas documentales proporcionan las herramientas esenciales y determinantes para responder a los objetivos formulados y llegar a resultados efectivos" (p. 58). Es decir, para responder con eficiencia a las necesidades investigativas, se introdujeron como técnica de recolección el método inductivo, que hizo posible 


\section{Estimulación temprana sinónimo de un mejor desarrollo infantil}

Vol. 3, núm. 1., (2019)

Eva Rossanna Ramos Miño; Sandra Dalila Pincay Cevallos; Gladys Lucia Llanos González; Celia Matilde Vinueza Villacis

llevar a cabo una valoración de los hechos de forma particular para llegar a la explicación desde una visión general.

Asimismo, se emplearon las técnicas de análisis de información para la realización de la investigación que fue ejecutada bajo la dinámica de aplicar diversos elementos encargados de determinar el camino a recorrer por el estudio, según, (Bolívar, 2015) las técnicas de procesamiento de datos en los estudios documentales "son las encargadas de ofrecer al investigador la visión o pasos que debe cumplir durante su ejercicio, cada una de ellas debe estar en correspondencia con el nivel a emplear" (p. 123). Esto indica, que para llevar a cabo el procesamiento de los datos obtenidos una vez aplicado las técnicas seleccionadas, tales como: fichas de resumen, textual, registros descriptivos entre otros, los mismos se deben ajustar al nivel que ha sido seleccionado.

\section{Resultados.}

El desarrollo infantil en los seres humanos se ve reflejado en las capacidades y destrezas que presentan en la edad de adolescente y adulta. Si los individuos son estimulados desde temprana edad se garantiza un adecuado crecimiento que ayude a impulsar los procesos mentales del cerebro del niño potenciando las funciones cognitivas, lingüísticas, motriz y social.

De este modo la creación de instituciones y centro tanto públicos como privados que lleven a cabo esta labor, es de suma importancia para la sociedad, más aun si se trata de países subdesarrollados, ya que, el avance de este tipo funciones va de la mano con el alcance de la población a la educación temprana promoviendo a las madres a continuar con la labor de estimular a sus hijos desde muy temprano en sus hogares guiadas por medio de planes y 


\section{Estimulación temprana sinónimo de un mejor desarrollo infantil}

Vol. 3, núm. 1., (2019)

Eva Rossanna Ramos Miño; Sandra Dalila Pincay Cevallos; Gladys Lucia Llanos González; Celia Matilde Vinueza Villacis

programas que se encarguen de explicar los ejercicios y juegos adecuados en cada etapa de los niños.

El Fondo de las Naciones Unidas para la Infancia o como siglas en ingles (UNICEF, 2011) indica que los ejercicios que se emplean desde los primeros días de nacido hasta los 5 años deben ser adecuados según la edad del infante. Adicional a esto, la revista digital para profesionales de la enseñanza en su publicación en el año 2011 expresa, que al inicio las actividades se enfocan en reforzar el vínculo emocional, masajes y estímulos sensoriales, respetando el desarrollo natural del bebé, y el instinto natural de sus padres. Luego se inician actividades de motricidad gruesa, motricidad fina, concentración y lenguaje. Es muy importante cuidar y proteger la iniciativa, la independencia y la autoestima del niño durante todo su proceso de aprendizaje. Al mismo tiempo vale la pena tomar en cuenta factores importantes para lograr aprovechar los estímulos adecuados a los cuales nuestros hijos/as pueden estar expuestos/as.

Entre las áreas de desarrollo se encuentran: el motor grueso que son los grandes movimientos del cuerpo, piernas y brazos. El motor fino que son los movimientos finos y precisos de las manos y dedos. El lenguaje que es la capacidad de comunicarse y hablar y el área socio-afectivo que es la capacidad de relacionarse con los demás y expresar sentimientos y emociones (UNICEF, 2011).

De acuerdo a la (UNICEF, 2011) las actividades se dividen según la edad de los niños, a continuación se hace mención a una serie de ejercicios y actividades que ayudan a estimular a los niños en el rango de 0 a 5 años de vida: 


\section{Estimulación temprana sinónimo de un mejor desarrollo infantil}

Vol. 3, núm. 1., (2019)

Eva Rossanna Ramos Miño; Sandra Dalila Pincay Cevallos; Gladys Lucia Llanos González; Celia Matilde Vinueza Villacis

- De 0 a 3 meses: Actividades para levantar y controlar la cabeza, actividades durante la alimentación y la lactancia materna que estimulen a la succión como tocar sus mejillas. Actividades para los brazos y piernas que consiste en mover los miembros de arriba abajo con la finalidad de mejorar el desarrollo motriz. Actividades para la visión y la audición que consisten en llevar al niño a sitios de diferente iluminación y colocar sonidos en sus oídos para llamar la atención. Actividades que tienen que ver con el lenguaje donde se recomienda imitar todos los sonidos que hace el bebe para incentivarlos a seguir haciéndolos.

- De 4 a 6 meses: En estos meses se trabaja la estimulación del motor grueso y el motor fino específicamente con actividades que los impulse a sentarse y a levantar el tronco con sus brazos cuando están boca abajo. Colócale juguetes en las manos y muévelo para llamar la atención del bebe, incentívalo a aplaudir y coloca objetos con consistencias de diferentes y estimula a tocarlos. Imita los sonidos que hace de balbuceo o gritos y háblale llamándole por su nombre con tonos suaves de voz.

- De 7 a 9 meses: Coloca al bebe en el suelo e incentiva el gateo por medio de juguetes y objetos que llamen su atención, juega con él para que quiera agarrarlos y de esta manera comience a gatear. Deja que coma solo y experimente la textura de los alimentos y la coordinación de las manos al comer.

- De 10 a 1 año: Estimula los movimientos que hace el bebe para intentar pararse, colócalo en sitios donde se pueda sujetar, enséñalo a pararse y a sentarse solo, ayúdalo a 


\section{Estimulación temprana sinónimo de un mejor desarrollo infantil}

Vol. 3, núm. 1., (2019)

Eva Rossanna Ramos Miño; Sandra Dalila Pincay Cevallos; Gladys Lucia Llanos González; Celia Matilde Vinueza Villacis

dar pasos agarrado de objetos o de las manos de un adulto. Llévalo a jugar con otros niños para que interactúe y socialice.

- De 1 año a 1 año y medio: En este rango de edad el bebe está apto para pararse a jugar con el juguete de su preferencia, enséñalo a agacharse y levantarse solo, muéstrale como recoger y guardar los juguetes que están en el suelo, permite que haga sonidos con objetos sonoros como por ejemplo una cuchara con cualquier objeto metálico, enséñalo a hacer torres con cubos o bloques de juguete, enséñale los nombres de las partes del cuerpo, pídele que traiga objetos que son de atención y aplaude sus logros, canta junto a él o ella canciones infantiles y motívalo a bailarlas, deja que interactúe con otros niños y enséñalo a compartir, explícale como se organiza la familia y deja que tome decisiones propias para que se sienta involucrado en casa.

- De 1 año y medio a 2 años: Aplica juegos en casa que sienta que lo persigues y enséñalo a patear pelota, enséñalo a subir y bajar de sitios altos a otros más bajos como escaleras y muros, estimula con una hoja de papel y lápiz y deja que raye libremente, muéstrale como tapar y destapar frascos plásticos y a arrugar papel en bolitas, pregúntale si quiere ir al baño y comienza a enséñalo a controlar los esfínteres, anímalo a vestirse solo y a ayudar en actividades de casa como limpiar guardar u ordenar.

- De 2 a 3 años: Enséñale a pararse de puntilllas y a levantarse del suelo sin usar las manos, enséñalo a saltar de un lugar a otro y sobre obstáculos y muéstrale como pararse en un solo pie, responde cada pregunta que te haga e indícale las diferencias entre niñas y niños, enséñalo a bajarse la ropa para ir al baño, incentiva la limpieza y muéstrale 


\section{Estimulación temprana sinónimo de un mejor desarrollo infantil}

Vol. 3, núm. 1., (2019)

Eva Rossanna Ramos Miño; Sandra Dalila Pincay Cevallos; Gladys Lucia Llanos González; Celia Matilde Vinueza Villacis

como hacer tareas sencillas como recoger juguetes y ayudar en actividades de casa, ayúdalo a que comprenda el sentido de pertenencia de manera feliz, pregúntale recurrentemente como se siente y ayúdalo a identificar sus sentimientos.

- De 3 a 4 años: Enséñale como caminar de puntillas, juega a atrapar pelotas en el aire, incentívalo con juegos en donde tenga que saltar, correr, caminar y que deba detenerse de repente, muéstrale canciones en donde tenga que coordinar movimientos, con papel y lápiz incentívalo a dibujar libremente y pregunta acerca de las cosas que dibuja, muéstrale como armar rompe cabezas, cultiva su imaginación ayudándolo a dibujar y crear historias acerca de lo que dibuja, a esta edad permite que vaya solo al baño, enséñalo a vestirse desvestirse solo y déjalo escoger como quiere vestirse.

- De 4 a 5 años: Incentiva juegos de correr saltar y caminar, enséñalo a hacer maromas con cuidado déjalo caminar en una línea recta y muéstrale como se hace, enséñalo a caminar sin zapatos de puntillas y con el talón, continua animándolo a recortar dibujar y colorear y enséñalo a hacerlo cada vez mejor, muéstrale como vaciar agua de un recipiente a otro sin derramarlo, pregúntale acerca de lo que hace y le gusta hacer, enséñale los números y a diferenciar entre derecha e izquierda, enséñale adivinanzas sencillas, pregúntale a diario como le va en la escuela e interactúa con las maestras para reforzar lo que aprendió en el salón de clases, llévalo a jugar con otros niños, explícale cuando hace algo mal y felicítalo cuando hace cosas positivas. 


\section{Estimulación temprana sinónimo de un mejor desarrollo infantil}

Vol. 3, núm. 1., (2019)

Eva Rossanna Ramos Miño; Sandra Dalila Pincay Cevallos; Gladys Lucia Llanos González; Celia Matilde Vinueza Villacis

Sin embargo, hay factores que van ligados al éxito que se pueda tener durante el desarrollo infantil, uno de los más importantes es la nutrición representando el punto clave en el éxito de la estimulación temprana y dejando en evidencia que los niños mal nutridos no desarrollan de igual manera las funciones cognitivas, lingüísticas, motriz y social que un niño que mantiene una alimentación adecuada según su edad.

La autora (Paris, 2011) en su publicación acerca del coeficiente intelectual en la adolescencia mostró, que para el 2004 se llevó a cabo un estudio con adolescentes de edades comprendidas entre 12 y 16 años, al que se les escaneó las estructuras cerebrales con resonancia magnética y se les sometió a tests de inteligencia. Cuatro años más tarde, al repetir las mismas pruebas, detectaron que el coeficiente intelectual había variado en los jóvenes. Se observaron cambios en la estructura cerebral, y que el CI aumentaba en unos casos y disminuía en otros (hasta 20 puntos de diferencia). Los autores señalan que la inteligencia de los niños se sigue desarrollando y puede incluso mejorar hasta mucho más tarde. Sin embargo, la directora del estudio expone que es posible que las diferencias se deban a la estimulación temprana o tardía que tuvieron los niños estudiados.

Esto se debe a que los primeros años de vida son fundamentales para el aprendizaje, ya que, es cuando se da una migración masiva de células a la corteza cerebral, aumentando la capacidad de almacenaje de información e incrementando la memoria y la capacidad de aprender. De ahí la importancia de la estimulación temprana. 


\section{Estimulación temprana sinónimo de un mejor desarrollo infantil \\ Vol. 3, núm. 1., (2019) \\ Eva Rossanna Ramos Miño; Sandra Dalila Pincay Cevallos; Gladys Lucia Llanos González; Celia Matilde Vinueza Villacis}

\section{Discusión y conclusiones}

Es evidente que la estimulación infantil representa un tema de mucha atención en padres y cuidadores de niños y que debe ser empleado desde su nacimiento con el fin de impulsar las funciones cognitivas, lingüísticas, motriz y social, considerando la estimulación desde los primeros días de nacido,

Durante el desarrollo del proceso investigativo se observó, que procesos mentales del cerebro del niño se desarrollan desde muy temprana edad, por tal motivo se debe incentivar la estimulación para ayudar a los niños a desarrollarse más adecuadamente en todos las áreas necesarias a medida que van creciendo.

De acuerdo a esto se tiene, que para el desarrollo y el éxito en la estimulación se deben tener en consideración algunos factores de riesgo que retrasan este desarrollo como lo es la nutrición o el estado económico de la familia del infante, por tal motivo se observa la necesidad de implementar programas tanto públicos como privados que brinde a todo tipo de público el acceso a programas y centros para ser guiados logrando enseñar a estimular de manera temprana a sus bebes.

Las actividades que se mencionan dentro de la investigación están enfocadas en el desarrollo del motor grueso, el motor fino, el lenguaje y el área socio-afectivo de los niños, estas actividades están caracterizadas por juegos y tareas que se realizan con el bebe desde que tienen 0 edad hasta los 5 años de vida evidenciando el aporte que proporciona en el desarrollo del niño y los beneficios que esto trae en la edad adolescente y adulta de la persona. 


\section{Estimulación temprana sinónimo de un mejor desarrollo infantil}

Vol. 3, núm. 1., (2019)

Eva Rossanna Ramos Miño; Sandra Dalila Pincay Cevallos; Gladys Lucia Llanos González; Celia Matilde Vinueza Villacis

En la investigación también se observó, que el coeficiente intelectual en adolescentes está ligado a la estimulación que se ha dado a temprana edad, un estudio realizado y mencionado revela que el CI aumenta o disminuye con el crecimiento de los individuos producto de la estimulación que estas personas han tenido en sus primeros años de vida.

Finalmente se puede concluir, que la estimulación temprana es la base para el desarrollo de todas las áreas y funciones de los seres humanos, que a través, de una estimulación temprana se pueden lograr seres con un mayor coeficiente intelectual y que esto también depende de las condiciones económicas de los infantes, siendo sumamente importante la combinación de la estimulación con una nutrición adecuada para reforzar las funciones cognitivas y de lenguaje de cada individuo así como también la motricidad y la interacción social.

\section{Bibliografía.}

Bolívar, J. (2015). Investigación Documental. México. Pax.

Castro, J. (2016). Técnicas Documentales. México. Limusa.

Dávila, A. (2015). Diccionario de Términos Científicos. Caracas: Editorial Oasis.

Heckman, J. \&. (15 de Febrero de 2007). The productivity argument for investing in young children. Recuperado el 28 de Abril de 2019, de Downloaded from: http://jenni.uchicago.edu/human

Heckman, J. S. (2006). The Effects of Cognitive and Noncognitive Abilities on Labor Market Outcomes and Social Behavior. Journal of Labor Economics, 24-411.

Paris, E. (8 de Noviembre de 2011). Bebeymas.com. Recuperado el 28 de Abril de 2019, de https://www.bebesymas.com/desarrollo/la-estimulacion-temprana-podria-hacer-variar-elcociente-intelectual-en-la-adolescencia

UNICEF. (Diciembre de 2011). UNICEF. Recuperado el 28 de Abril de 2019, de http://files.unicef.org/mexico/spanish/ejercicioestimulaciontemprana.pdf 


\section{Estimulación temprana sinónimo de un mejor desarrollo infantil}

Vol. 3, núm. 1., (2019)

Eva Rossanna Ramos Miño; Sandra Dalila Pincay Cevallos; Gladys Lucia Llanos González; Celia Matilde Vinueza Villacis

Wachs, T. (2000). Necessary but not sufficient: the role of individual and multiple influences on human development. American Psychological Association Press. Washington D.C. 